\section{COMUNICACIONES ORALES}

\section{ANÁLISIS DE LOS CUIDADOS EN LOS ÚLTIMOS DÍAS DE VIDA DE LOS PACIENTES HOSPITALIZADOS EN MEDICINA INTERNA}

R. Gómez-Méndez (1), R. Suárez-Gil (1) M. F. Liroa-Romero (1), V. Álvarez-Vidal (2), A. García-Martínez(3), R. Rabuñal-Rey (4)

HOSPITAL UNIVERSITARIO LUCUS AUGUSTI. 1: MEDICINA INTERNA, 2: GERIATRÍA, 3: CUIDADOS PALIATIVOS, 4: UNIDAD DE ENFERMEDADES INFECCIOSAS

INTRODUCCIÓN: Existen muy pocos estudios centrados en las actuaciones en los últimos días de vida de pacientes hospitalizados en fase terminal de su enfermedad. OBJETIVOS. Analizar las intervenciones sobre pacientes fallecidos en planta de Medicina Interna en los que la muerte era esperable.

MATERIAL Y MÉTODO: Estudio descriptivo, retrospectivo de pacientes fallecidos en Medicina Interna en Enero-Agosto de 2017. Se excluyeron pacientes fallecidos antes de la valoración por un internista y aquéllos con muerte no esperable. Definimos muerte esperable como aquélla en la que el médico responsable registró en la historia clínica la posibilidad del fallecimiento. Se analizaron variables epidemiológicas, clínicas, diagnóstico-terapéuticas y evolutivas.

RESULTADOS: Se incluyeron 234 pacientes. La edad media fue de 85,5 años, el 50\% eran mujeres y la estancia media fue de 8,8 días. El 66,7\% tenía un índice de Charlson $\geq 7$. La causa más frecuente de muerte fue infección respiratoria $(21,8 \%)$.

Se registraron los síntomas en el $97,4 \%$, la información a familiares sobre mal pronóstico en el $91 \%$, la orden de no RCP en el 33,3\%; sólo constaba el documento de instrucciones previas en 1 paciente.

El $66,2 \%$ recibió tratamiento paliativo: $49 \%$ pauta fija+ rescate, $38,9 \%$ rescates y $12,1 \%$ pauta fija. Se retiró tratamiento no sintomático en el 27,5\% y se inició sedación en el 56,4\%, siendo ésta indicada por el médico responsable en el $85,1 \%$ de los casos. El consentimiento sobre sedación constaba en la historia clínica en el $54,7 \%$ de los casos. Se intentó RCP en $1,7 \%$ y se realizaron procedimientos diagnóstico-terapéuticos no sintomáticos en el 32,5\%. CONCLUSIONES: La mayoría de pacientes fallecidos nuestra planta en los que la muerte era esperable son ancianos pluripatológicos. Es necesario mejorar el abordaje del paciente paliativo en Medicina Interna, tanto desde el punto de vista de la planificación como de la intervención terapéutica.

\section{ATIPIA TIROIDEA DE SIGNIFICADO INCIERTO. RIESGO DE MALIGNIDAD}

Isabel Fernández Castro. Cristina Trigo Barros. Ruth Boente Varela. José Luis Lamas Ferreiro. Wilfredo Guanipa. Manuel de Sas Fojón.

POVISA. MEDICINA INTERNA YENDOCRINOLOGIA

INTRODUCCIÓN: El sistema Bethesda clasifica la atipia de significado incierto en la categoría III, y la prevalencia de malignidad de esta es muy dispar entre los distintos estudios, con un rango que varía entre el 5 y el 37\%. Por este motivo existe discrepancia entre el manejo de estos nódulos, bien repetir PAAF o derivar directamente a cirugía. El objetivo de este trabajo es analizar el riesgo de malignidad en nuestra área sanitaria para determinar la actitud clínica más adecuada. MATERIAL Y MÉTODO: desde mayo de 2010 a marzo de 2017 se analizaron 1229 citologías tiroideas, de las cuales 99 fueron clasificadas en la categoría III del sistema Bethesda, un $8 \%$. Analizamos el resultado de los pacientes en los que se repitió la PAAF, el resultado de los operados y correlacionamos las características ecográficas con el riesgo de malignidad.

RESULTADOS: en 11 pacientes (11.1\%) se hizo seguimiento. En 30 (30.3\%) se repitió la PAAF: $15(50 \%)$ resultaron benignas, 6 (20\%) insuficientes, 7 (23.3\%) atipia de significado incierto y 2 $(6.76 \%)$ sospechosas de malignidad. Se operaron 67 pacientes, 56 se mandaron directamente y 11 se remitieron tras la segunda PAAF. De estos, $33(49.2 \%)$ fueron benignas y $34(50.7 \%)$ malignas: 9 (26.5\%) microcarcinoma papilar de tiroides, 1 (2.9\%) carcinoma medular, $1(2.9 \%)$ carcinoma papilar bien diferenciado con potencial maligno incierto, 6 (17.6\%) carcinoma papila variante folicular y 17 (50\%) carcinoma papilar. Ninguna característica ecográfica tuvo significación estadística como factor de riesgo de malignidad, sin embargo, una edad inferior a 50 años sí. CONCLUSIÓN: de las 99 citologías informadas como atipia de significado incierto, el 34.4\% mostraron cáncer de tiroides tras la cirugía. Ante estos resultados, debido al alto porcentaje de malignidad en esta categoría, consideramos que lo adecuado es recomendar tiroidectomía a todos los pacientes clasificados como categoría III, pese a discrepar con las recomendaciones del sistema Bethesda.

\section{PUESTA EN MARCHA DE UN PROGRAMA DE TRATAMIENTO DE INSUFICIENCIA CARDÍACA REFRACTARIA CON DIÁLISIS PERITONEAL}

Pedrosa Fraga C (1), Guerrero Sande H (1), Cerqueiro González JM (1), Piñeiro Fernández JC (1), González Tabarés L (2), Millán Díaz B (2) HOSPITAL UNIVERSITARIO LUCUS AUGUSTI. (1)MEDICINA INTERNA (2)NEFROLOGÍA

INTRODUCCIÓN: La insuficiencia cardiaca refractaria (ICR) es un problema de salud con elevada morbimortalidad. La mayoría de pacientes presentan enfermedad renal crónica (ERC), lo que incrementa la estancia hospitalaria, asistencia a urgencias y rescates en hospitales de día. El síndrome cardiorrenal asocia resistencia a diuréticos, por lo que hace necesaria la búsqueda de alternativas terapéuticas.
OBJETIVOS: Evaluar la eficacia de la diálisis peritoneal (DP) en el tratamiento de ICR en cuanto a calidad de vida, hospitalización y mortalidad.

MATERIAL Y MÉTODO: Estudio descriptivo retrospectivo de los pacientes con ICR incluidos en el programa de DP entre enero de 2014 y abril de 2018. Se analizó la mejoría en la calidad de vida, asistencias al hospital, mortalidad y seguridad.

RESULTADOS: Se incluyeron 11 pacientes (9 varones): 7 con ICR por cardiopatía isquémica, 2 por miocardiopatía dilatada y 2 por cardiopatía valvular. La edad media fue de 81, 8 años. Todos presentaban ERC estadio 3b-5 (ninguno en diálisis) y precisaron asistencia a urgencias, ingreso o rescates en hospital de día en el año previo al inicio de DP, por insuficiencia cardíaca (IC), con una mediana de 29,36 (4-62) días-año. Todos destacaron la mejoría en su calidad de vida. Sólo 2 precisaron ingreso por IC (estancia media 8 días) y uno asistencia a urgencias. Ninguno precisó rescates de diuréticos iv, paracentesis ni toracocentesis. 3 presentaron peritonitis por DP (resueltas sin complicaciones). 6 (54,5\%) fueron exitus: 1 por edema agudo de pulmón, 4 por deterioro progresivo tras una media de 13,5 meses en DP y 1 por fractura de cadera. Ningún paciente falleció por causas derivadas de DP. 5 pacientes continúan en el programa, con un tiempo medio de seguimiento de 21,91 (4-82,5) meses.

CONCLUSIONES: La DP mejora el control de la sobrecarga de volumen, disminuye las hospitalizaciones y mejora la calidad de vida de los pacientes. La adaptación a la técnica es buena y con escasas complicaciones.

\section{ESCLEROSIS SISTÉMICA Y CÁNCER. POSIBLE PAPEL DE LOS CALCIOANTAGONISTAS}

Andrea Cabaleiro, Mayka Freire, Omayra Rodríguez, Mila Suárez, Antón Otero, Olalla Lima, Patricia Diéquez, Ana Argibay, Caritina Vázquez, Alberto Rivera

COMPIEXO HOSPITALARIO UNIVERSITARIO DEVIGO. UNIDAD DE ENFERMEDADES AUTOINMUNES SISTÉMICASY TROMBOSIS. SERVICIO DE MEDICINA INTERNA

INTRODUCCIÓN: El uso de calcioantagonistas (CA) está muy extendido en la esclerosis sistémica (ES) para el tratamiento del Fenómeno de Raynaud. Los CA pueden alterar la apoptosis, un mecanismo para la destrucción de células cancerosas. En la ES, el riesgo de cáncer aumenta, pero el papel de los CA no está claro, con datos contradictorios hasta el momento

OBJETIVOS: Analizar el papel de los CA en la aparición de cáncer en una cohorte de pacientes con ES.

MATERIAL Y MÉTODO: Se recogieron pacientes diagnosticados de ES en nuestro hospital desde 1985 hasta 2017. Se revisaron las historias clínicas, registrando los datos clínico-epidemiológicos y los tratamientos.

RESULTADOS: 120 pacientes fueron diagnosticados de ES (103 mujeres, 17 hombres), 22 de los cuales (18,3\%) han desarrollado cáncer. El tipo de cáncer fue: mama (12 pacientes, $57 \%$ ), gastrointestinal (4 pacientes, 33\%), un hipernefroma, un carcinoma endometrial, un linfoma, un cáncer de piel y un epidermoide de lengua. El adenocarcinoma fue el patrón histológico más frecuente (16 pacientes, $76 \%$ ). El diagnóstico de ES se realizó a una edad más avanzada en los pacientes diagnosticados de cáncer (66,4 +/- 11,7 vs $54,8+/-17,3$ años, $p=0,019)$; Una edad mayor de 55 años confería un riesgo relativo (RR) de cáncer de 1,69 (1,26-2,25) en la cohorte global y un RR de 3,26 (1,18-8,99) en la cohorte femenina. A pesar de que la asociación entre cáncer y CA no alcanza significación estadística, la asociación entre CA y pacientes mujeres mayores de 55 años eleva el RR de cáncer a 4,16 (0.96-17,95). Todas las demás características analizadas no alcanzaron diferencias estadísticamente significativas.

CONCLUSIONES: Los CA aumentan, en nuestra serie, el riesgo de cáncer en mujeres mayores de 55 años. Los CA podrían estar implicados en la patogenia del cáncer en la ES. Debido al amplio uso de estos fármacos en la ES, sería interesante aclarar mejor dicha asociación con estudios controlados de mayor tamaño.

\section{CORRELACIÓN DE FÓRMULAS PARA ESTIMACIÓN DE FILTRADO GLOMERULAR PARA AJUSTE DE DOSIS DE ANTICOAGULANTES DE ACCIÓN DIRECTA EN UNA COHORTE DE PACIENTES DE MEDICINA INTERNA}

P. Vázquez Rodríguez, R. Suárez Fuentetaja, A. B. Porto Pérez, I. Rodríguez Osorio, I. Justo Muradás, C. Barbagelata López, S. Ruanova Suárez, R. De La Fuente Cid, J. L. Díaz Díaz COMPLEXO HOSPITALARIO UNIVERSTTARIO A CORUÑA. MEDICINA INTERNA

INTRODUCCIÓN: Anticoagulantes acción directa(ACOD)son alternativa eficaz y segura frente acenocumarol en pacientes con fibrilación auricular no valvular (FANV); de elección según quías práctica clínica(GPC). 1/3 pacientes con FANV tienen enfermedad renal crónica (ERC) al menos moderada, pudiendo ser medida con fórmulas como CKD-EPI, MDRD, Cockrolf-Gault (CG). Ensayos pivotales de ACOD incluyeron en torno $20 \%$ pacientes con ERC ajustando dosis según ACOD y filtrado glomerular estimado(FGe)mediante fórmula CG. Así, GPC recomiendan medir FGe mediante $C G$ en pacientes con FANV que van a recibir $A C O D$. En nuestra red sanitaria se usa mayoritariamente CKD-EPI. 2-Conocer concordancia entre CG y CKD-EPI para cálculo FGe que obliga a ajuste de dosis de ACOD en FANV. Calcular valor predictivo positivo(VPP)CKD-EPI para dicho fin 3-Estudio de cohortes retrospectivo en $>75$ años ingresados en un Servicio de Medicina Interna(MI)con FANV y CHADS-VASC $\geq 2$. Para cálculo FGe se tomó media de valores de creatinina en últimos 6 meses, excluyendo ingreso hospitalario. 4-Características basales poblacionales $(\mathrm{N}=306$ ) se muestran en Tabla1. \%pacientes con ERC al menos moderada es parecido cuando se calculó con CKD-EPI (78,2\%) o CG (74.9\%), con coeficiente correlación kappa entres ambas fórmulas 0.77 para todo el espectro de FGe (Gráfica1). Aplicando fórmula CG deberíamos ajustar dosis de rivaroxaban o edoxaban en $57.5 \%$ pacientes, $62.4 \%$ si usamos CKD-EPI cuyo VPP para tal fin es $64 \%$ (Gráfica3). \% para apixaban son 14.7 y $14.4 \%$ respectivamente, y VPP para CKD-EP de 58\% 5-3/4 partes de pacientes ingresados en $\mathrm{Ml}>75$ años con FANV tienen ERC al menos moderada con independencia de fórmula con que midamos FGe(CG o CKD-EPI), mostrándose buena correlación entre ambas fórmulas. De usarse CKD-EPI para cálculo FGe en esta población, estaríamos modificando dosis ACOD en 40\% pacientes en los que el cálculo mediante CG no nos obligaría a ello. El uso CKD-EPI para valoración FGe en esta población puede conducir a infradosificación de ACOD 


\section{FACTORES PREDICTIVOS DE DIAGNÓSTICO DE TUBERCULOSIS PULMONAR EN URGENCIAS HOSPITALARIAS}

lago Aldao, Ana Chouza, Nagore Blanco, Ángeles Pallarés, Laura Calviño,

Ángel Salgado, Luis Anibarro

COMPLEXO HOSPITALARIO UNIVERSITARIO DE PONTEVEDRA. FACULTAD DE MEDICINA USC. INSTITUTO DE INVESTIGACIÓN SANITARIA GALICIA SUR. UNIDAD DE TUBERCULOSIS-MEDICINA INTERNA. NEUMOLOGIA.

MICROBIOLOGIAA

INTRODUCCIÓN: La tuberculosis pulmonar (TBP) es una enfermedad de potencial transmisión nosocomial a partir de pacientes no aislados a su ingreso. Por otra parte, un sobreaislamiento provoca sobrecostes y disminución innecesaria de la disponibilidad de camas.

OBJETIVOS: Realización de un score predictivo que identifique la necesidad de aislamiento respiratorio de pacientes en Urgencias con sospecha de TBP que precisen ingreso.

MATERIAL Y MÉTODO: Estudio de casos y controles de pacientes ingresados con sospecha de TBP en el Complexo Hospitalario de Pontevedra entre 2011 y 2016. La sospecha de TBP se basó en la solicitud de cultivos de micobacterias en muestras respiratorias. Se comparó cada caso con dos controles seleccionados de las solicitudes inmediatamente anterior y posterior. Se analizaron las variables disponibles en el servicio de Urgencias.

Tras un análisis preliminar de los datos, se realizó un modelo de regresión logística múltiple con las variables potencialmente predictoras. Se asignó una puntuación a cada variable en base a los valores Exp $(\beta)$ y el valor de significación. Posteriormente se evaluó la curva ROC para detectar el mejor punto de corte de discriminación.

RESULTADOS: Se incluyeron 207 pacientes: 69 casos y 138 controles. Las variables predictoras de diagnóstico final de TBP fueron: la exposición previa conocida a TB $(p=0$, 023), sintomatología constitucional $(p=0,002)$, fiebre de $\geq 2$ semanas $(p=0,046)$, ausencia de disnea $(p=0,001)$, afectación radiológica en lóbulos superiores $(p=0,002)$ con cavitación $(p=0,002)$, hiponatremia $(p=0,000)$ y ausencia de neutrofilia $(p=0,006)$. Con las variables predictoras se elaboró un score (Tabla 1). El AUC de la curva ROC fue 0,905 (IC95\%: 0, 861-0, 949), con sensibilidades y especificidades que se muestran en la Tabla 2. Un score $\geq 4$ presenta $90 \%$ de sensibilidad y $70 \%$ de especificidad.

CONCLUSIÓN: Un score $\geq 4$ en Urgencias indica la necesidad de aislamiento respiratorio.

\section{IMPACTO DE UN PROGRAMA DE VIGILANCIA DE ANTIBIÓTICOS RESTRINGIDOS SOBRE EL CONSUMO DE ANTIBIÓTICOS DE AMPLIO ESPECTRO, COSTES, ESTANCIA Y MORTALIDAD}

Jose Luis Lamas Ferreiro (1), Ana Sanjurjo Rivo (1), Judith Álvarez Otero (1), Isabel Fernández Castro (1), Fernando Maroto Piñeiro (1), Jose Carlos de Miguel Bouzas (2), Jose Ramón Bermúdez Sanjurio (1), Javier de la Fuente Aguado (1)

HOSPITAL POVISA. (1) SERVICIO DE MEDICINA INTERNA (2) SERVIIIO DE FARMACIA

INTRODUCCIÓN: El objetivo fue evaluar el impacto de un programa de vigilancia de antibióticos restringidos. Características del programa: con una base de datos electrónica se identifican diariamente los pacientes en tratamiento con antibióticos restringidos y se revisan las historias de los que han recibido 3 días de tratamiento. Se discuten con el médico responsable las alternativas terapéuticas.

MATERIAL Y MÉTODO: Se revisaron retrospectivamente las acciones realizadas en pacientes con prescripciones de antibióticos restringidos entre Nov-2016 y Ago-2017. Se compararon los datos de consumo con el período de Nov-2015 y Ago-2016. Se evaluó la diferencia en dosis diarias definidas (DDD) por 100 estancias de los antibióticos analizados y la diferencia en el gasto en antibióticos restringidos. Se calculó la mortalidad global en ambos períodos. Se comparó la mortalidad a 30 días y estancia media del grupo de pacientes evaluados por el programa con respecto al grupo en los que se prescribieron antibióticos restringidos en el mismo período pero que no pudieron ser revisados por sobrecarga asistencial. RESULTADOS: se revisaron 431 prescripciones, suspendiéndose el 24, 8\%. 352 prescripciones no pudieron ser evaluadas por sobrecarga asistencial. El programa supuso una reducción en DDD/100 estancias del 13,2\%. Fl ahorro en costes (euros/100 estancias) de antibióticos restringidos fue del $18,3 \%$ (20431 euros). No hubo diferencias significativas en la mortalidad entre períodos (diferencia de mortalidad entre períodos de $0,3 \%$ ) ni en mortalidad o estancias comparando los pacientes evaluados por el programa con los que no se pudieron revisar (diferencia de mortalidad entre grupos de $0,8 \%, P=0,8$; diferencia de estancia media de 4,7 días, $P=0,3$ ).

CONCLUSIONES: el desarrollo de un sistema de vigilancia de antibióticos restringidos es una herramienta eficaz para disminuir el consumo de antibioterapia de amplio espectro sin impacto sobre la mortalidad y estancia media de los pacientes. 